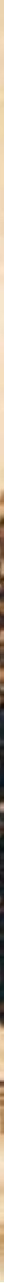

\title{
Die Familie Schlenker und ihre Rittergüter bei Torgau
}

Weßnig, neues und altes Herrenhaus, nach 1902

(c) Heimatmuseum Bennewitz, Fotosammlung

\section{Sigrun Jäschke}

\section{Die Nachfahren von}

\section{Friedrich Mathias Schlenker}

Die Nachfahren des im 19. Jahrhundert aus Württemberg zugewanderten Friedrich Mathias Schlenker bewirtschafteten zwischen 1879 und 1945 mehrere Rittergüter in der Umgebung Torgaus. Anhand aktueller genealogischer Forschungen soll ihr Schicksal vorgestellt werden.

\section{Friedrich Mathias Schlenker}

Friedrich Mathias Schlenker wurde 1800 als zweiter Sohn in Talheim (Württemberg) geboren. Seine Vorfahren gehören einem bekannten, schon damals weit verzweigten Familienclan aus der Gegend um Schwenningen an. Nach seiner Lehre im Brauerei- und Mälzerhandwerk in Tuttlingen arbeitete er während etlicher Brauereisaisonen auf der Wanderschaft in „Baiern und Würtenberg“" und schließlich auch in Sachsen. Nach der großen Völkerschlacht gegen Napoleon 1813 verschlug es ihn um 1823 nach Pegau in die südliche Umgebung von Leipzig. Dort zeugte er mit der Brauereibesitzerstochter Johanne Friedericke Löscher aus Pegau einen Sohn, der am 27. Februar 1825 getauft wurde. ${ }^{2}$ Der finanzielle Ausgleich zum gegebenen Eheversprechen und der Kindesunterhalt mussten bei ihm eingeklagt werden, wie eine 1826 angelegte Akte zum „Ehe- und Schwängerungsanspruch“ der Brauereibesitzerstochter belegt. ${ }^{3}$ In Pegau hatte der junge Friedrich Mathias Schlenker mindestens eine Brauereisaison gearbeitet, bevor er in den nahen Ort Liebertwolkwitz weiterzog. „Nach dem Eidbuch von Liebertwolkwitz leistete der damals 24 Jahre alte Friedrich Mathias Schlenker am 9. Mai 1825 den Brauer- und Mälzereid und wurde danach als Brauer und Mälzer der Commun-Brauerei verpflichtet."4

Eine weitere amtliche Akte behandelt nach dem Verkauf der Commun-Brauerei von Liebertwolkwitz, an der er sicherlich durch seine Heirat mit der Ratskellerwirtin Christiane Herbst ${ }^{5}$ Anteilseigner geworden war, am 29. November 1836 den Antrag „auf Anlegung und Betreibung einer Bier-
Danksagung

Die Autorin dankt ihrem Neffen und allen weiteren angeheirateten Familienmitgliedern für die Überlassung von Kopien und Fotos, aber auch für das Vertrauen, ihr Interna zur Familiengeschichte zu erzählen. Viele Erzählungen des Vetters Theoderich Schlenker in ihren Kindheitstagen haben nun einen Sinn bekommen. Ferner gedankt sei vor allem Wolfgang Lehmann, dem Besitzer des liebevoll gestalteten Fahrrad- und Heimatmuseums in Bennewitz, der uns viele seiner Dokumente und Fotos scannen ließ, und den Einwohnern aus Ammelgoßwitz, Weßnig und Bennewitz für ihre bereitwillige Auskunft über die Familie Schlenker. Zu danken ist auch Birgit Richter vom Staatsarchiv Leipzig, Fritz Halm aus Leipzig, der über die Brauereigeschichte Leipzigs forscht, und "Artsch" von ahnenforschung. net für Dokumentenbeschaffungen und Hinweise. 


\section{Stammtafel Friedr.-M. Schlenker}

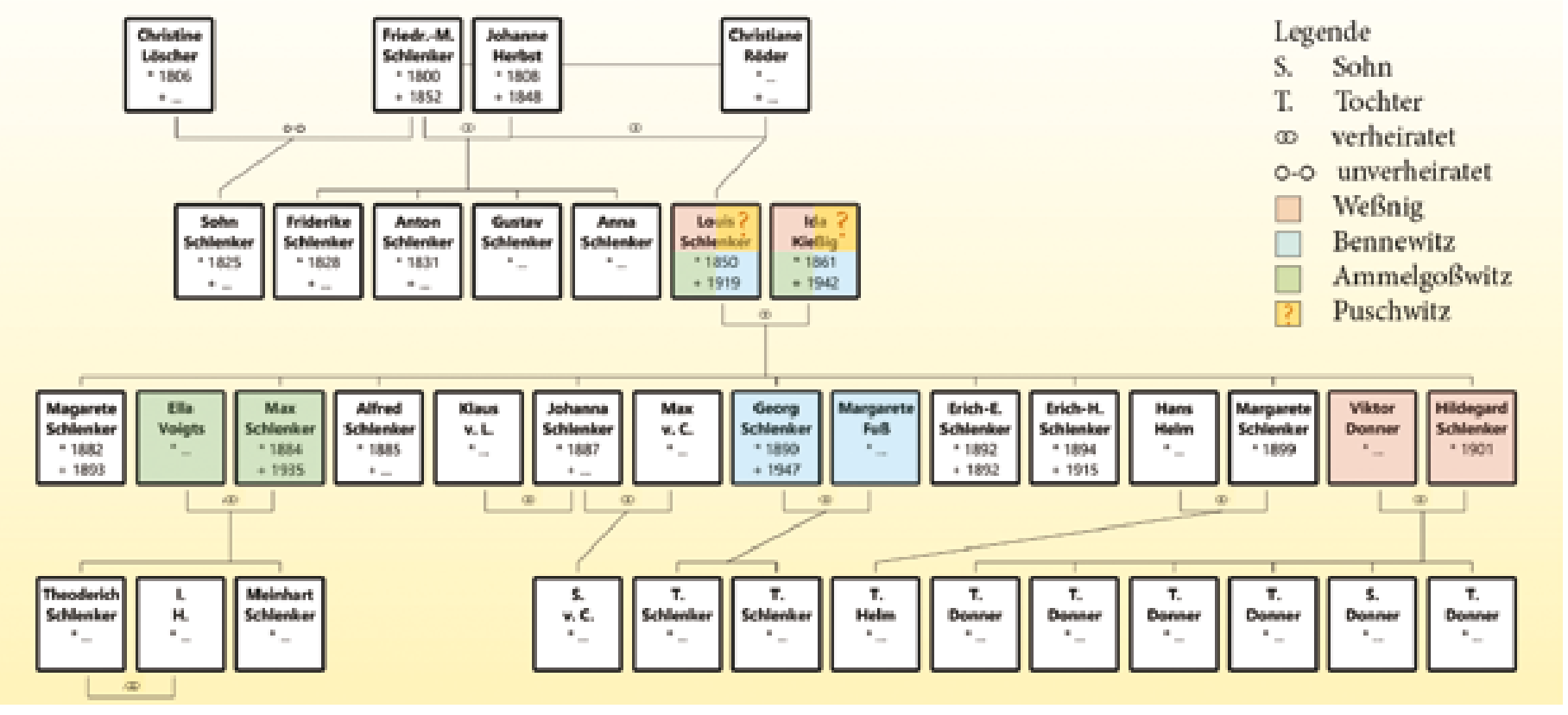

Stammtafel der Familie Schlenker bis 1945

1 Sächsisches Staatsarchiv, Staatsarchiv Leipzig (folgend StA Leipzig), 20460 Rittergut Liebertwolkwitz (Patrimonialgricht), Nr. 126.

2 StA Leipzig, 20021 Konsistorium Leipzig, Nr. 2207.

3 Ebenda.

4 Zitiert nach Fritz Halm, in StA Leipzig, 20460 Rittergut Liebertwolkwitz (Patrimonialgricht), Nr. 111.

5 Theoderich Schlenker: Von der Schusterkugel zum Rittergut. In: Das Heimatblättle (Villingen-Schwenningen) 27 (1979), Heft 11, S. 1-4.

6 Wie Anm. 1

7 Theodor Voigt: Liebertwolkwitz in den Tagen der Schlacht bei Leipzig am 14.18. October 1823 nebst einer kurzen Chronik der Parochie und Beschreibung der am 19. Oktober 1847 auf dem Monarchenhügel stattgefundenen Feier. Leipzig 1848.

8 Schlenker 1979 (wie Anm. 5). brauerei in Liebertwolkwitz“6. Dieses Gesuch wurde abgelehnt, da der vormalige Pächter und nun neue alleinige Besitzer der Commun-Brauerei, Johann Friedrich Liebner, am 5. September 1836 diese unter der Vorgabe ersteigert hatte, dass er keine weitere Konkurrenz im Ort bekomme. Die Commun-Brauerei war somit privatisiert. Schlenker ging jedoch davon aus, dass, obwohl er Profiteur durch den Verkauf seiner Anteile an der Brauerei war, seine Brauereirechte nicht erloschen seien.

Dass sich Friedrich Mathias Schlenker in Liebertwolkwitz erfolgreich emporarbeitete und in der Bevölkerung zu Ansehen gelangte, belegt seine mehrmalige Wiederwahl als Gemeindevorstand ab dem Jahre $1847 .{ }^{7}$ Leider muss jedoch die Familienüberlieferung, dass er 1850 in Liebertwolkwitz die Gründung der ersten Sparkasse veranlasst habe ${ }^{8}$, korrigiert werden. Aber es geschah wohl in seiner Zeit als Gemeindevorstand. Die Ehre gebührt Friedrich Teichmann, dem zum Gedenken 50 Jahre später ein Denkmal neben dem Rathaus, in dem die ersten Sparkassenräume untergebracht waren, enthüllt worden ist.

Im Jahr 1848 musste Friedrich Mathias Schlenker einen großen familiären Verlust hinnehmen. An nervösem Fieber starben erst im Juli sein 15-jähriger Sohn Anton, der Schüler der höheren Bürger- und Realschule zu Leipzig war, und dann am 16. August seine 39-jährige erste Frau Johanne Christiane und seine 19-jährige Tochter Friederike Wilhelmine.9 Anton wird bei der am 19. Oktober 1847 stattfindenden Enthüllung des ersten Denkmales der Völkerschlacht auf dem sogenannten Monarchenhügel bei Liebertwolkwitz als Altist der Schulknaben erwähnt. ${ }^{10}$

Um 1849 heiratete Friedrich Mathias Schlenker die Gutserbin Charlotte Röder. Zu seinen weiteren Kin- dern aus erster Ehe, Gustav und Anna ${ }^{11}$, wurde er am 19. April 1850 noch einmal Vater von Sohn Louis. Doch bereits zwei Jahre später am 16. März 1852 wurde der Junge durch den Tod seines Vaters Halbwaise. Hier verliert sich die Spur zu den älteren Geschwistern in Liebertwolkwitz und vorerst auch zu Louis.

\section{Louis Schlenker}

Die Familienüberlieferung besagt, dass Louis Schlenker Verwalter adliger Güter wurde und auch kräftig als erster Schnitter in der Kolonne die Sense führte. 1879 wurde er Pächter des Ritterguts Weßnig und heiratet 1881 Ida Kiessig, Tochter eines Rittergutsbesitzers aus Zabeltitz. 1902 kauften beide das Rittergut Weßnig und 1904 das Rittergut Bennewitz. ${ }^{12} 1902$ waren sie bereits Eltern von neun Kindern, von denen Ida Margarete zehnjährig eine Operation nicht überstand, Ernst Erich als Säugling starb und Hans Erich später im Ersten Weltkrieg fiel. ${ }^{13}$

1910 erwarb Louis Schlenker noch das Kirchengut Ammelgoßwitz I und das Rittergut Ammelgoßwitz II. Somit konnten Louis Schlenker und seine Frau Ida ihre Söhne später mit Grundbesitz versorgen. Das Rittergut Puschwitz wird bei Donath und Schlenker ebenfalls als Eigentum der Familie Schlenker erwähnt, war aber bis 1945 wohl in Verwaltung, Pacht oder Besitz der Familie Hermann Karl Neubürger und dessen Sohn Martin. ${ }^{14}$ Hier besteht noch Forschungsbedarf. Bekannt ist, dass Max und Ella Schlenker sehr eng mit der Familie Neubürger befreundet waren. Louis Schlenker ließ 1905 ein neues Herrenhaus in Weßnig bauen, das Bennewitzer Schloss ausbauen und errichtete 1910 das Herrenhaus in Ammelgoßwitz II neu, dessen Vorgängerbau 1890 abgebrannt war. 


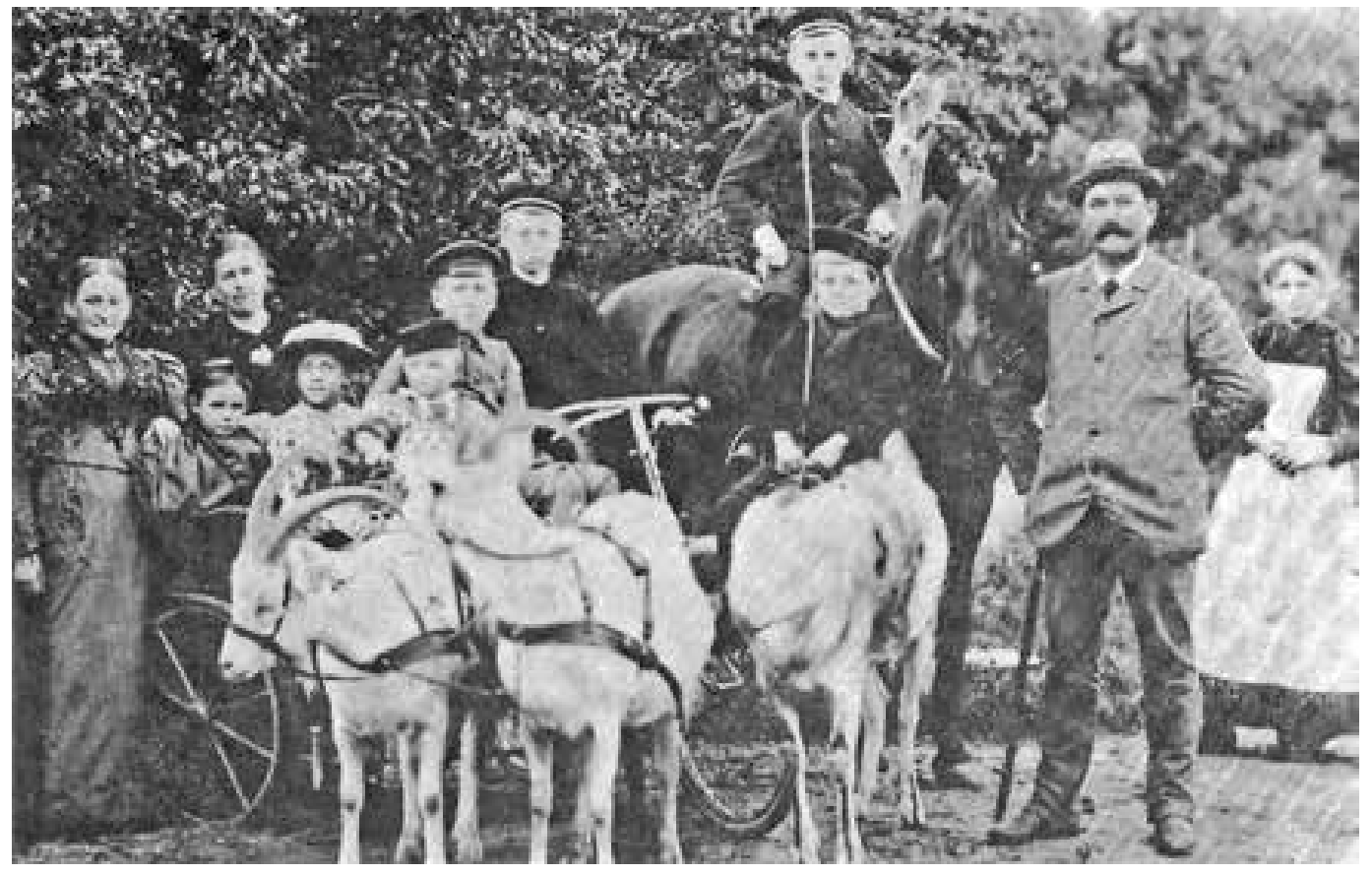

Louis Schlenker muss ein tüchtiger, umtriebiger und angesehener Gutsherr gewesen sein, der wohl auch sein reichliches väter- und mütterliches Erbe gut angelegt hatte. Er arbeitete sich zum königlich preußischen Ökonomierat empor und wurde stellvertretender Landrat in Torgau. Er und seine Frau kümmerten sich verantwortungsvoll um ihre Angestellten und ebenso um „ihre“ Dörfer, wie es später auch deren Kinder und Schwiegerkinder taten. Noch heute sprechen die Dorfbewohner bzw. die Nachkommen der Angestellten gut über „ihre“ Schlenker-Familie und können mit vielen Erzählungen und Berichten aus ihren Familien aufwarten.

„Mit dem Kauf des Rittergutes Weßnig übernahm Louis Schlenker auch das Patronat über die Ortskirche. Das Ehepaar stiftete der Kirche zwei Buntglasfenster"15, von denen heute nur noch das rechte neben dem Altar erhalten ist.

Die Kinder von Ida und Louis Schlenker heirateten Offiziere und/oder Rittergutsbesitzer und Töchter aus Rittergütern, die diese bei entsprechenden Veranstaltungen in ihren Kreisen kennenlernten, so auf dem „Rübenball“ in Halle.

Max Schlenker und Ammelgoßwitz

Der älteste Sohn Max Schlenker „studierte bilden-

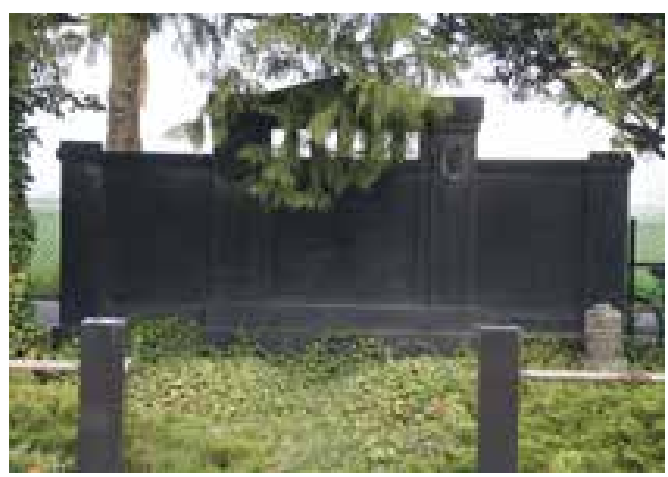

de Kunst an der Académie Francaise“ in Paris ${ }^{16}$ und legte als leidenschaftlicher Dendrologe teten teilweise 200 Landarbeiter gleichzeitig“17. Sein Ammelgoßwitzer Herrenhaus verwandelte er „mit Kunstsammlungen bedeutender Künstler des 16. und 17. Jahrhunderts zu einem ,mon bijou', das zum Anziehungspunkt schöngeistiger Leute aus aller Welt wurde“18. Max Schlenker starb 1935.

Seine Frau Ella, die Max Schlenker auf einer Landwirtschaftsausstellung in Braunschweig kennengelernt hatte, bewirtschaftete bis zum Kriegsende 1945 das Rittergut mit Hilfe eines Verwalters. Ihren polnischen Kriegsgefangenen behandelte sie jedenfalls gut. Dieser bewahrte sie nach Kriegsende vor dem Erschießen, so dass sie auf ihren väterlichen Hof nach Meerdorf bei Braunschweig fliehen konnte. Alle Kunstwerke von Max Schlenker und auch die von staatlichen Stellen eingelagerten Kulturgüter wurden von der sowjetischen Besatzungsmacht beschlagnahmt.

Ella und ihr erstgeborener Sohn Theoderich vermissten schon ein wenig das Leben als Rittergutsbesitzer, wo Theoderich Schlenker als Kriegsversehrter auch ganz anders hätte weiter wirtschaften können. Er bekam zu DDR-Zeiten jahrelang bei seinen Besuchen in Ammelgoßwitz immer mal

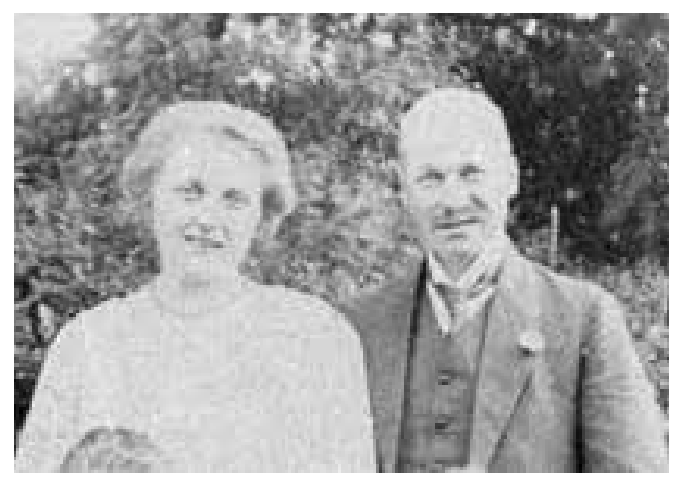
1927/1928 den Treblitzscher Park an. Hier ,arbei-
Louis Schlenker und seine Familie um 1902 Foto: Theoderich Schlenker

9 Todesanzeige Anton Schlenker in der Leipziger Zeitung vom 2. August 1848, Todesanzeige Johanne und Fridericke W. Schlenker in der Leipziger Zeitung vom 29. August 1848. Dank an Artsch vom forum.ahnenforschung.net für das Abschreiben der Anzeigen und weitere Hinweise.

10 Wie Anm. 7

11 Danksagung zum Tod von Friedrich Mathias Schlenker in der Leipziger Zeitung vom 23. März 1852.

12 Gustav Paul Schräpler: Geschichte der Parochie Weßnig. Weßnig 1904.

13 Schlenker 1979 (wie Anm. 5). 14 Matthias Donath: Schlösser und Herrenhäuser in Nordsachsen. Dresden 2014; Schlenker 1979 (wie Anm. 5). 15 Schräpler 1904 (wie Anm. 12). 16 Schlenker 1979 (wie Anm. 5). 17 Hans Köhler/Volker Bergstädt: Der Landschaftspark Treblitzsch. Ein dendrologischer Führer mit Gehölzverzeichnis und Erläuterungen sowie zwei Plänen. o.O. 1994.

links: Grabstätte der Familie Schlenker auf dem Friedhof von Weßnig

Foto: Uwe Ulrich Jäschke

rechts: Ella und Max Schlenker um 1920

๑ Fotosammlung Sigrun Jäschke 
18 Schlenker 1979 (wie Anm. 5).

19 Uwe Niedersen: Das Blech ist bis $\mathrm{zu}$ einem Zentimeter stark. In: Torgauer Zeitung vom 9. Dezember 2011.

20 Matthias Donath (Hrsg.): Die Erfindung des Junkers. Die Bodenreform 1945 in Sachsen. Meißen 2011.
Bennewitzer Angestellten dürfen sich mit einem schicken Auto ablichten lassen. ๑ Heimatmuseum Bennewitz, Fotosammlung wieder etwas aus dem Haushalt seiner Eltern zugesteckt, das er dann in den Westen schmuggelte. Ein Kuriosum sind „die zwei Festungstorflügel aus $1 \mathrm{~cm}$ dickem Bessemer Stahlblech, die Louis Schlenker nach der Schleifung der Festung Torgau (1889 vollendet) um 1900 erwarb und später als Brandschutztür mitten in die Ammelgoßwitzer Scheune einbauen ließ. Diese wurden nach der Wende vom Sachsen-Preußen-Kollegium des Fördervereins Europa Begegnungen e.V. geborgen und restauriert. Sie wurden 2011 im Brückenkopfgelände in der Nähe der dortigen Poterne von 1812 aufgestellt" ${ }^{* 19}$.

\section{Alfred Schlenker}

Sohn Alfred Schlenker, der gesundheitlich ein wenig das Sorgenkind der Familie war, kümmerte sich als Faktotum über viele Jahre um seine Mutter Ida und blieb nach Kriegsende allein in Weßnig zurück.

\section{Johanna Schlenker}

Tochter Johanna Schlenker war zweimal verheiratet und lebte nach Kriegsende in West-Berlin.

\section{Georg Schlenker und Bennewitz}

Sohn Georg Schlenker war im Ersten Weltkrieg ein erfolgreicher Jagdflieger und betrieb sein Bennewitzer Rittergut mit großen Fischteichen und einer Schnapsbrennerei mit Hilfe eines Verwalters. Die Fischteiche ließ er modernisieren und sorgte durch Teilung eines Teiches für eine bessere Wasserqualität.

Als leidenschaftlicher Flieger war er Mitorganisator von Flugtagen 1926 in Torgau und 1931 in Bennewitz. Stargast war die Fliegerin Elly Beinhorn. Das beeindruckende Flugprogramm mit Flugakrobatik ist in heutiger Zeit aus sicherheitstechnischen Gründen nicht mehr denkbar.

Im Rahmen der Enteignung der Rittergutsbesitzer wurde Georg Schlenker im Oktober 1945 vom Feld

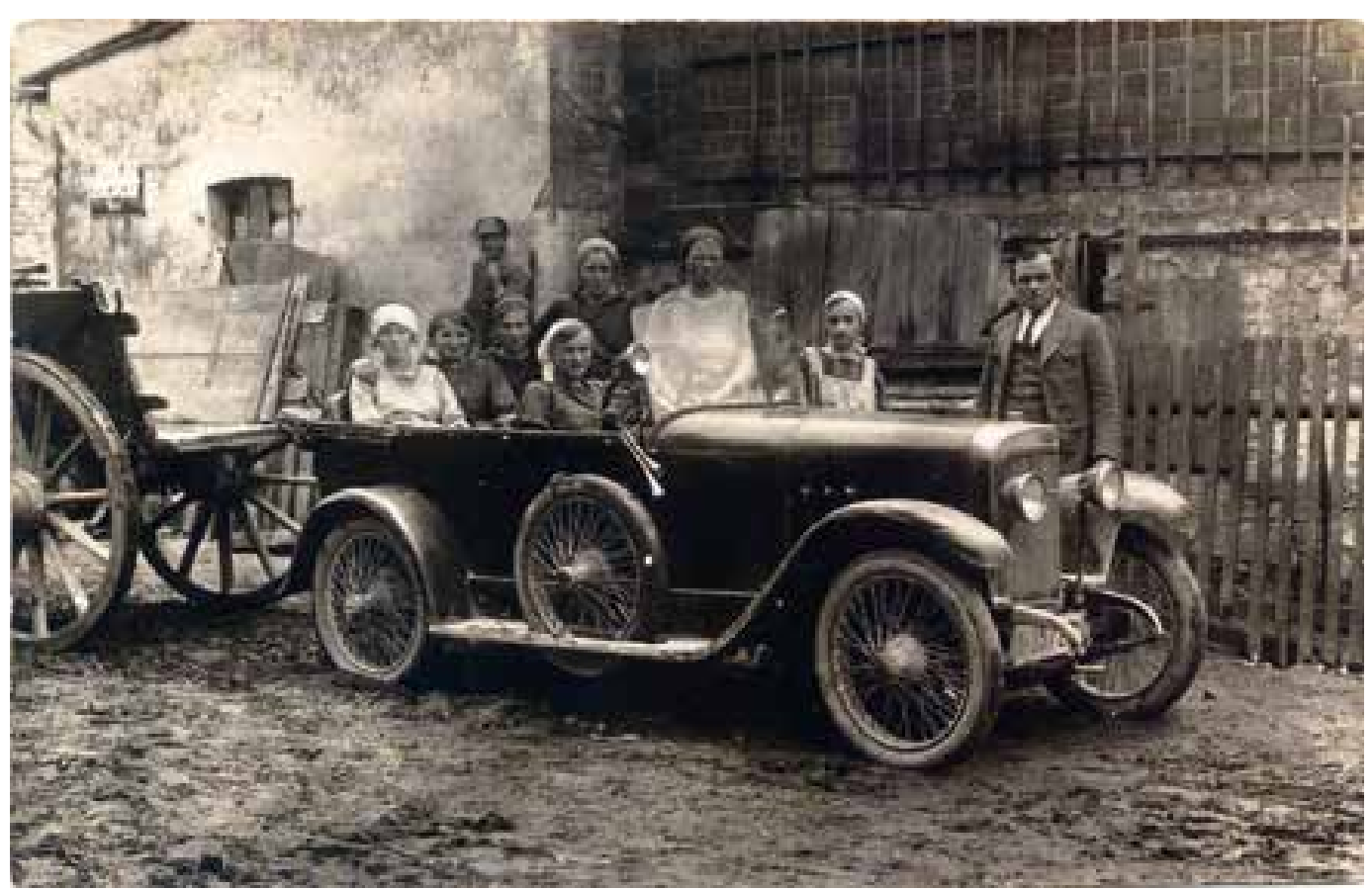

weg nach Torgau gebracht. Von dort aus kam er in das sowjetische Speziallager in Buchenwald, wo er 1947 starb. Seine Familie erfuhr erst nach seinem Tod von einem aus Buchenwald entlassenen Mithäftling von seinem Schicksal.

Seine Frau Margarete, genannt Marga, konnte noch mit Unterstützung ihrer beiden Töchter Teile des Mobiliars zu ihrer Schwägerin nach Meerdorf (Niedersachsen) retten. Außerdem standen zahlreiche Kisten bei ihren ehemaligen Angestellten unter, deren Inhalt sie nach und nach abholte. Eine ihrer Töchter blieb bei der Verwandtschaft ihres Mannes in Leipzig, die andere heiratete in den Raum Braunschweig.

\section{Margarete Schlenker}

Tochter Margarete Schlenker war mit Hans Helm, einem Offizier und Rittergutsbesitzer aus Dürschnisierte im Januar 1945, als die Sowjetische Armee im Osten Deutschlands vorrückte, einen großen Treck von Schlesien nach Weßnig. Hier machten sie erst einmal drei Monate Station, bevor sie in die englische Besatzungszone weiterzogen.

\section{Hildegard Schlenker und Weßnig}

Tochter Hildegard Schlenker erbte das Rittergut Weßnig, da ihr Bruder Hans Erich Schlenker im Ersten Weltkrieg gefallen war. Ihr Mann Viktor Donner, der anfangs als Verwalter auf dem Rittergut gearbeitet hatte, war ein guter und pragmatischer Landwirt. Er baute zum Beispiel eine Stammschäferei mit einem prämierten Bock, einem der besten Deutschlands, auf. Zeitweilig hatte Viktor Donner die Stelle des Kornmeisters in Torgau inne. Auch sorgten beide gut für ihre Angestellten. Sie erweiterten den Bestand der gutseigenen Wohnhäuser. Dank des besseren Bodens war das Gut Weßnig dem Gut Bennewitz immer einen witz bei Liegnitz/Schlesien verheiratet. Er orga- 


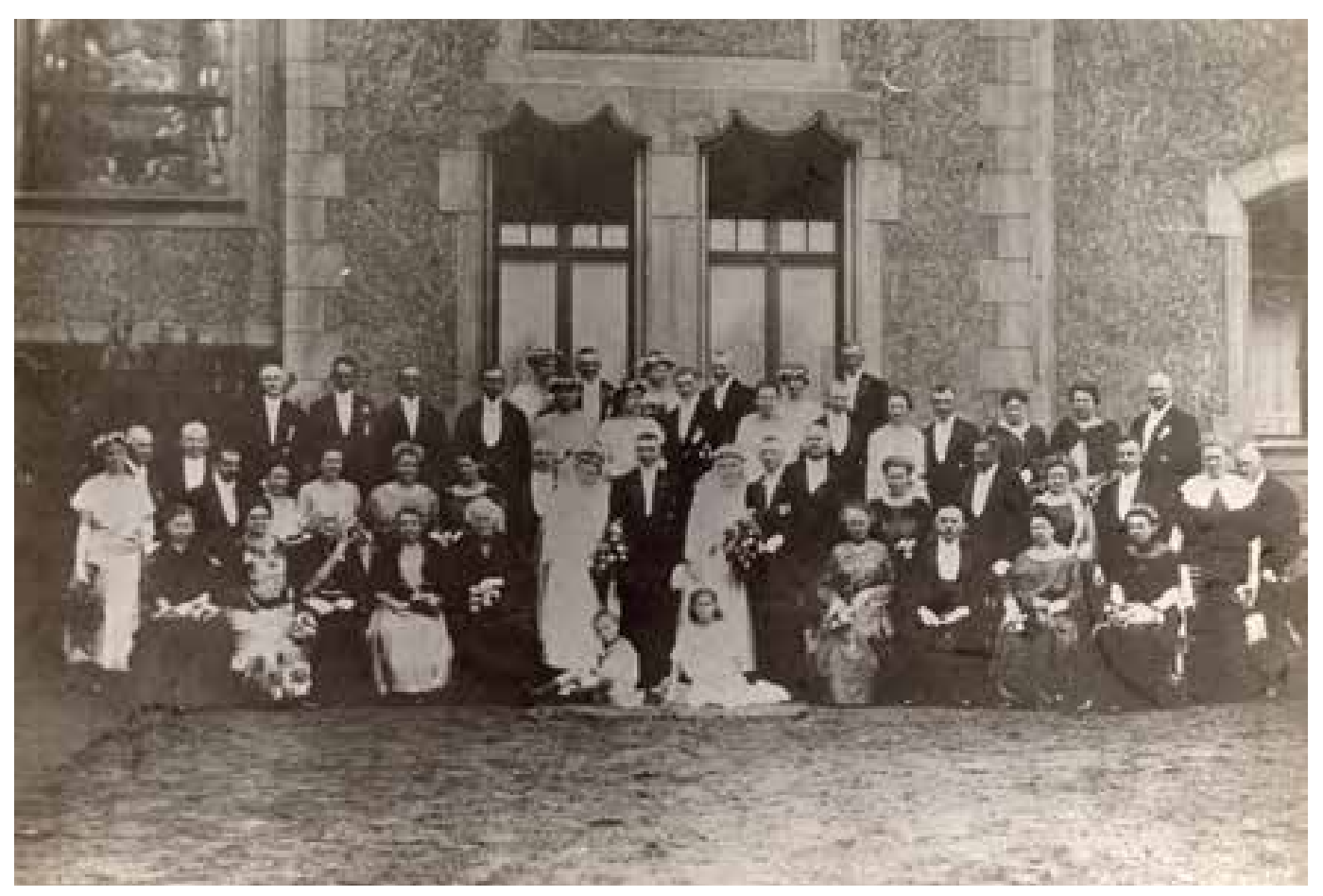

kleinen Schritt voraus. Bis Kriegsende schafften Hildegard und Viktor Donner es, das Rittergut Weßnig schuldenfrei zu bekommen, denn alle Hausneu- und umbauten, die Aussteuern und die Erbauszahlungen der Schwestern mussten größtenteils von hier erwirtschaftet werden.

Hildegard und ihrem Mann wurden im Zuge der Rittergutsenteignungen von einem Weßniger Ochsenhirten an einem Samstag im Oktober 1945 mitten während der Ernte mitgeteilt, dass die gesamte Familie mit Handgepäck binnen 24 Stunden den Landkreis zu verlassen habe, sonst würden sie nach Rügen verbracht werden. Sie packten daraufhin heimlich ihre Betten und wichtiges Hausgerät auf einen Ackerwagen, verließen durch einen Hinterausgang Weßnig und fuhren in der Nacht mit dem Traktor zu Verwandten nach Riesa. So etwas konnte nur mit Hilfe ihrer verschwiegenen Angestellten geschehen, denen Hildegard und Viktor Donner immer gute Arbeitgeber waren.

Mehrere Versuche, in Riesa, bei Oschatz, Cossebaude oder Leipzig wieder sesshaft zu werden, waren durch widrige Umstände nicht von langer Dauer, so dass sie Ende der 1960er Jahre zu ihrer Tochter nach Braunschweig übersiedelten. Ihre Kinder blieben teils in der DDR zurück oder flohen in den Westen. Eine Tochter wanderte über England in die USA aus. Nach den Wirren des Zweiten Weltkrieges und der Enteignung ihrer Güter haben alle Mitglieder der Familie Schlenker klein angefangen, um sich wieder eine bürgerliche Existenz aufzubauen.

Da die Familie Schlenker nach Kriegsende auf Anordnung der sowjetischen Militärverwaltung enteignet worden war, bekamen sie nach der Wende 1989 ihre Güter auf Grund des Einigungsvertrages von 1990 nicht zurück. ${ }^{20}$ Finanziell wurden sie auch nicht für ihren Grundbesitz entschädigt. Sie erhielten lediglich den Lastenausgleich, eine Nutzungsausfallentschädigung als Eingliederungshilfe, der in der
Bundesrepublik Deutschland für Enteignete aus dem Gebiet der DDR ab 1972 ausgezahlt wurde.

\section{Die Schlenker-Besitzungen}

\section{Weßnig}

1902 kaufte Louis Schlenker das 250 Hektar große Rittergut Weßnig. 1913 gehörten zum Gutsbetrieb 19 Pferde, 95 Rinder, 509 Schafe und 106 Schweine. Vor 1928 übernahm Viktor Donner, vorher Verwalter in Weßnig, das Rittergut. Er hatte 1923 Hildegard Schlenker geheiratet. 1928 umfasste das Gut 263 Hektar. Die Enteignung 1945 betraf Hildegard und Viktor Donner. Die Gutsgebäude werden bis heute als Schule und Hort genutzt. Die benachbarte Kirche ist seit 2003 die erste Radfahrerkirche Deutschlands.

\section{Bennewitz}

Das Rittergut Bennewitz wurde 1904 von Louis Schlenker gekauft, der das barocke Jagdschloss zu einem repräsentativen Wohnsitz ausbaute. 1913
Doppelhochzeit in Weßnig 1923

(c) Heimatmuseum Bennewitz,

Fotosammlung
Weßnig, neues und altes Herrenhaus, dahinter die Kirche Foto: Dr. Matthias Donath

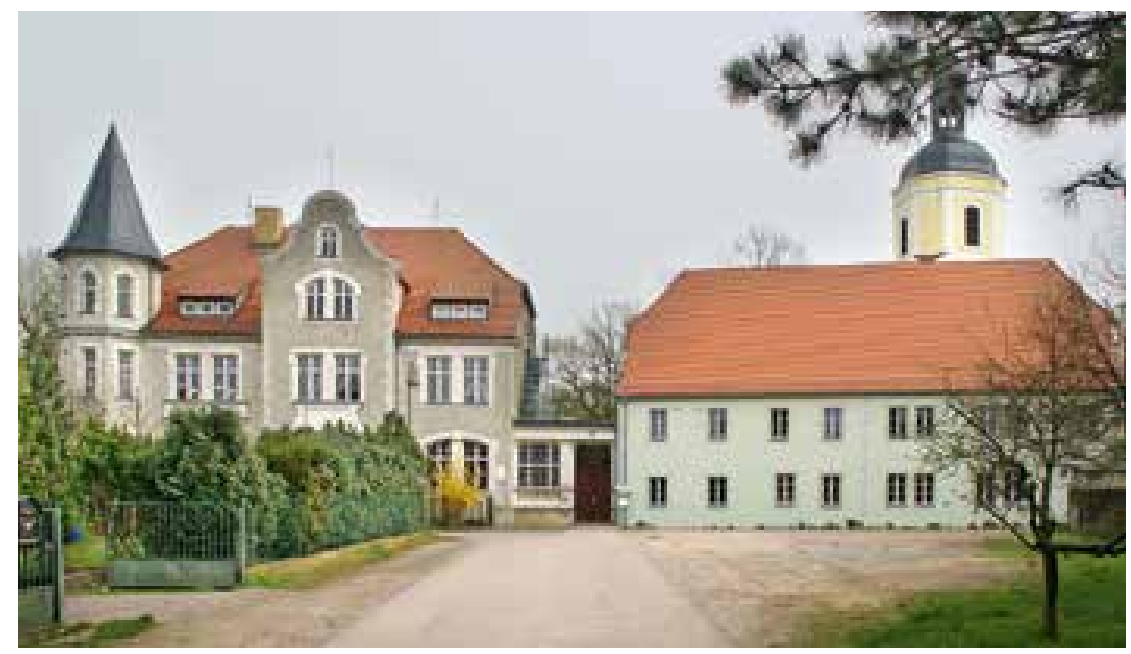




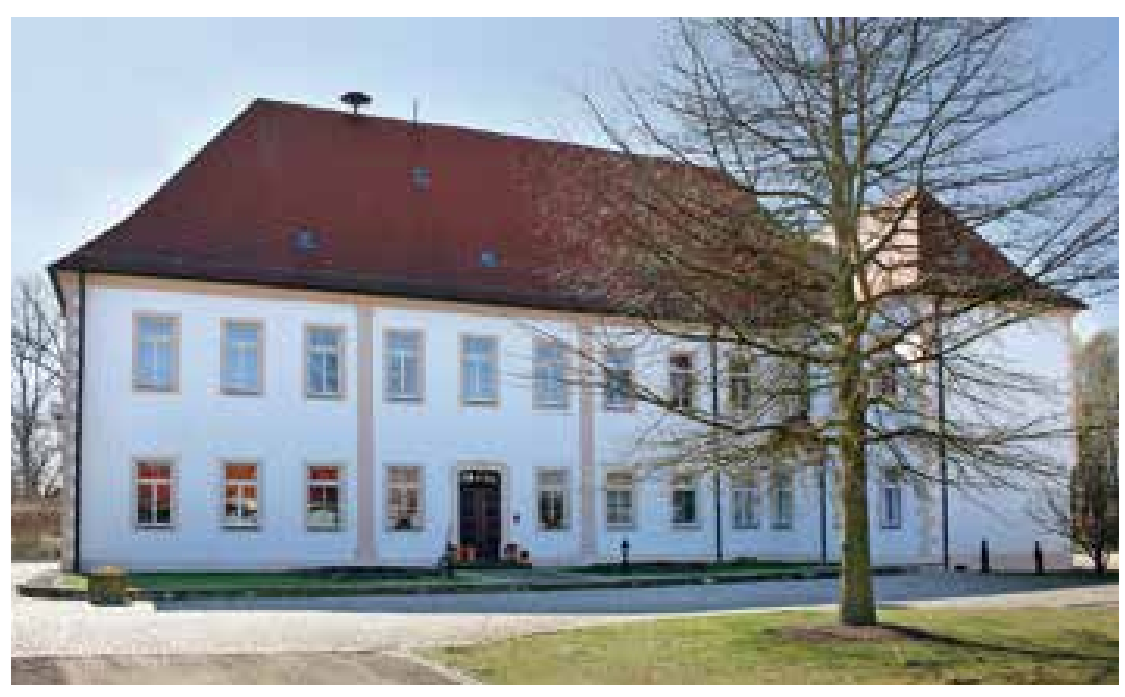

Herrenhaus Bennewitz Foto: Dr. Matthias Donath

rechts: Grabkreuz für Max und Meinhart Schlenker im Treblitzscher Park Foto: Sigrun Jäschke

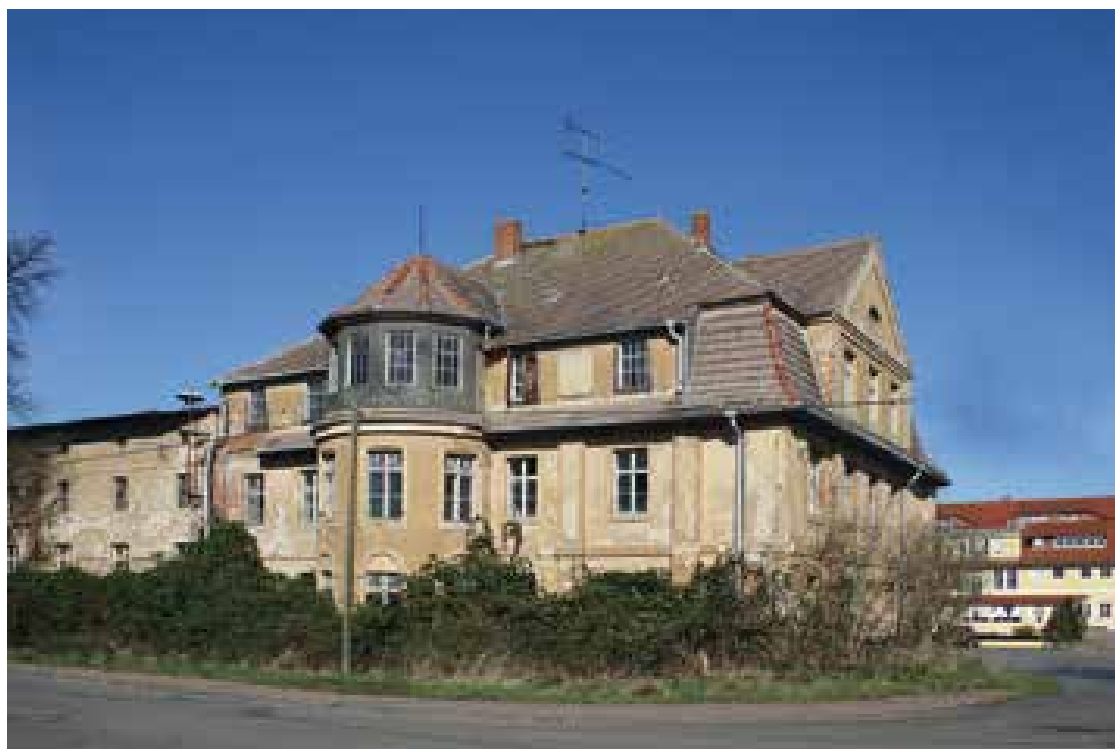

Herrenhaus des Ritterguts Ammelgoßwitz II Foto: Dr. Matthias Donath

Autorin

Sigrun Jäschke Dresden umfasste der Betrieb 346 Hektar Land, davon 283 Hektar Acker, 27 Hektar Wiesen und 21 Hektar Wald, Fischteiche, 17 Pferde, 53 Rinder und 35 Schweine. Vor 1928 übernahm Georg Schlenker das Rittergut. Dieser wurde 1945 enteignet. Aus dem Rittergut mit seinen Teichen ging die Bennewitzer Lehr- und Versuchsteichwirtschaft hervor. In den vergangenen Jahren wurde das Herrenhaus von einem Torgauer Unternehmer liebevoll saniert; es ist heute Firmensitz.

\section{Ammelgoßwitz I und II}

1910 kaufte Louis Schlenker die Güter Ammelgoßwitz I (Klostergut) und Ammelgoßwitz II (Rittergut). Das Herrenhaus Ammelgoßwitz II ist ein schlichter Barockbau. Das später von Max Schlenker bewohnte Gutshaus Ammelgoßwitz Ii wurde 1910 im neobarocken Stil neu erbaut. 1913 gehörten zu beiden Rittergütern 177 Hektar Land, davon 111 Hektar Acker, 37 Hektar Wiesen und 26 Bewirtschaftung Er kauft außerdem 1927 ein fünf Hektar großes Gelände, auf dem er den Treblitzscher Park anlegen ließ. 1945 wurde Ella Schlenker enteignet. Das Gutshaus Ammelgoßwitz II Hektar Wald. 1919 übernahm Max Schlenker die diente in der DDR-Zeit als Lehrlingswohnheim und steht heute leer.

\section{Treblitzscher Park}

Max Schlenker kaufte 1927 das 5 Hektar große Gelände, um daraus bis 1928 einen Landschaftspark mit Sichtachsen zu gestalten. Ca. $250 \mathrm{Ge}$ hölze wie Laub- und Nadelbäume und seltene Koniferen, außerdem Rhododendren, japanische Azaleen, Rosen und Magnolien sind bis heute zu bestaunen. Viele kleine Brücken über Bäche und Wege entlang von Teichen lockern die Parkfläche auf. 1935 wurde Max Schlenker in seinem Park beigesetzt. Daneben befindet sich das Scheingrab seines zweitgeborenen Sohnes Meinhart, der 1943 in der Ukraine fiel. Nach dem Zweiten Weltkrieg litt der Park unter dem Brennholzmangel der Bevölkerung, und die Wiese wurde als Fußballfeld missbraucht. Heute wird das Kleinod von der Universität Leipzig wissenschaftlich betreut.

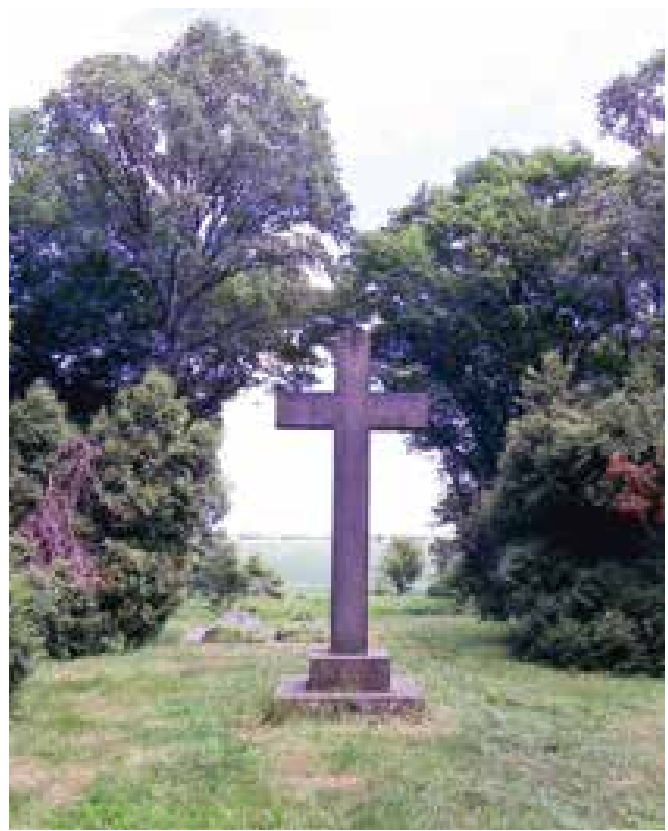

\section{Puschwitz}

Ob und in welcher Form das Rittergut Puschwitz im Eigentum der Familie Schlenker gewesen ist, konnte in der Kürze der Zeit nicht geklärt werden. Theoderich Schlenker schrieb, dass Louis Schlenker „Herr und Eigentümer der Rittergüter Weßnig, Bennewitz, Puschwitz sowie des Kloster- und Landgutes Ammelgoßwitz" wurde. 1913 wird indes Rittmeister der Reserve Hermann Karl Neubürger als Eigentümer des Ritterguts Puschwitz genannt. Das Rittergut, das möglicherweise den Lebensunterhalt von Alfred Schlenker sichern sollte, umfasste damals 176 Hektar Land, davon 158 Hektar Ackerland, 10 Hektar Wiesen und 7 Hektar Wald. 1945 wurden Hermann Karl Neubürger und sein Sohn Martin Neubürger enteignet. Das schlichte Herrenhaus stammt aus dem 18. Jahrhundert. Es harrt nach einer ersten Renovierung in den 1990er Jahren einer weiteren Nutzung. 